\title{
Negotiating Instruction Strategies during Robot Action Demonstration
}

\author{
Lars C. Jensen \\ Institute for Design and \\ Communication \\ University of Southern \\ Denmark \\ DK-6400 Sonderborg \\ larje09@student.sdu.dk
}

\author{
Kerstin Fischer \\ Institute for Design and \\ Communication \\ University of Southern \\ Denmark \\ DK-6400 Sonderborg \\ kerstin@sdu.dk
}

\author{
Dadhichi Shukla \\ Institute of Computer \\ Science \\ University of Innsbruck \\ AT-6020 Innsbruck \\ dadhichi.shukla \\ @uibk.ac.at
}

\author{
Justus Piater \\ Institute of Computer \\ Science \\ University of Innsbruck \\ AT-6020 Innsbruck \\ justus.piater \\ @uibk.ac.at
}

\begin{abstract}
This paper describes the kinds of strategies naïve users of an industrial robotic platform make use of and analyze how these strategies are adjusted based on the robot's feedback. The study shows that users' actions are contingent on the robot's response to such a degree that users will try out alternative instruction strategies if they do not see an effect in the robot within a time frame of two seconds. Thus, the timing of the robot's actions (or in-actions) influences how users instruct the robot.
\end{abstract}

\section{Categories and Subject Descriptors}

H.1.2 [User/Machine Systems]: human factors

\section{General Terms}

Human Factors

\section{Keywords}

robot action demonstration; intuitive instruction strategies; delay;

\section{INTRODUCTION}

This study addresses the strategies human users take intuitively when they demonstrate novel actions to a robot and how the effectiveness of these strategies is negotiated between user and robot based on the timing of the robot's responses. Understanding what strategies users intuitively take when instructing a robot is relevant not only for service robots, where naïve users may want to adapt robots to their particular domestic environments, but also in industrial settings where understanding what strategies are the most intuitive may help prevent errors when various employees may demonstrate novel actions to a robot. The current study therefore aims to determine first what strategies users intuitively start out with.Second, it traces how users modify their instruction strategies over the first minutes of the teaching interactions based on the robot's responses; that is, we analyze what effect the timing of the robot's response has on the choice of users' instruction strategies over time. Especially if robots are meant to adjust to human users, it is crucial to know how their behavior is adapted to the robot over time.

Permission to make digital or hard copies of part or all of this work for personal or classroom use is granted without fee provided that copies are not made or distributed for profit or commercial advantage and that copies bear this notice and the full citation on the first page. Copyrights for third-party components of this work must be honored. For all other uses, contact the Owner/Author.

Copyright is held by the owner/author(s).

HRI'15 Extended Abstracts, Mar 02-05 2015, Portland, OR, USA

ACM 978-1-4503-3318-4/15/03

http://dx.doi.org/10.1145/2701973.2702036

\section{LITERATURE REVIEW}

Much research addresses aspects of the timing of robot responses; especially in the area of social robotics, previous work shows consistently that delays influence the quality of interactions. For instance, Sidner et al. [1] shows that human communication partners judge a robot producing contingent non-verbal feedback to be more reliable and its movements more appropriate, and they interact longer with the robot and attend to it more. Moreover, Kose-Bagci et al. [2] find that human participants prefer interactions in which a humanoid robot's turn-taking behavior is driven by a contingency model whose temporal dynamics is the closest to 'natural' human-human conversations. Finally, experiments by Lohan et al. [3] and Fischer et al. [4] show that the timing of robot responses influences the extent to which users tutor the robot.A few studies furthermore address intuitive user behaviors in the tutoring of robots; for instance, Thomaz \& Breazeal [5] analyze users' intuitive tutoring strategies and find that users' intuitive non-verbal tutoring strategies during learning from demonstration facilitate robot learning [6]. However, Chernova \& Thomaz [7] in their recent review of robot learning from human teachers argue that users' behaviors in such tutoring situations have not been sufficiently studied; thus, what effects the timing of robot behaviors may have on the strategies human tutors choose is still open.

\section{METHOD}

We elicited 34 interactions in which users were free to tutor a robot in any way they thought suited. The robot's task is to assist the user during the assembly of an IKEA toolbox. The data investigated here are the first minutes of the interactions in which users negotiated a strategy for the subsequent tutoring.

\subsection{Procedure}

Participants were led into the lab, signed a consent form, had their picture taken and were then shown to the robot and told that the robot would be able to fetch the parts for them, but that they would need to instruct it to do so when appropriate and in whatever way that made sense to them; participants should then assemble the box on their own. Participants were led to believe that the robot acted autonomously, but was in fact controlled using the Wizard-of-Oz technique. The human 'wizard' was instructed to react to gestures (e.g. pointing) and to ignore all other actions by the participants (e.g. speech). The 'wizard' had multiple cameras to observe the participants and to navigate the workspace. Due to the intranet, there was a slight delay for about one second between users' instruction and robot response. 


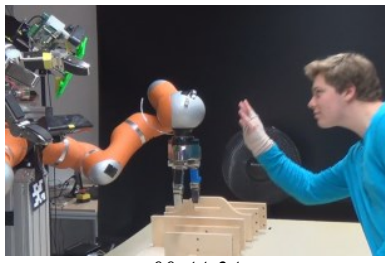

$00: 44: 24$ 5: And keeps pointing

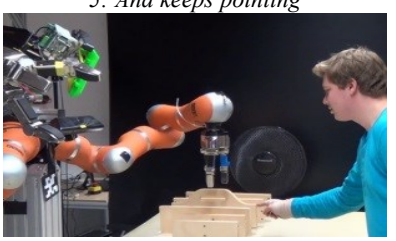

$00: 39: 15$

2: Points to board

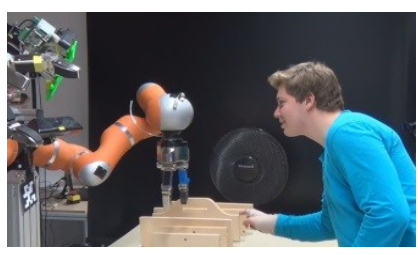

$00: 47: 08$

6: Moves hand downwards

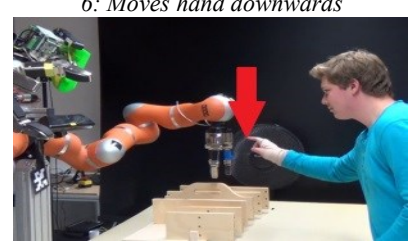

$00: 41: 11$

3: "Robot, I need this one" Pinches the board

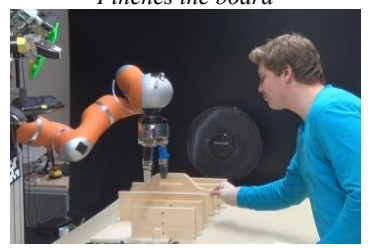

00:49:19

7: Pinches fingers together

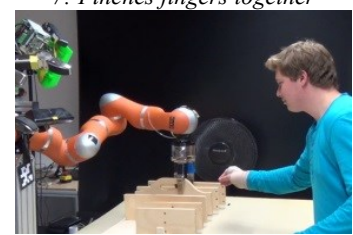

$00: 43: 23$

4: Points to board again

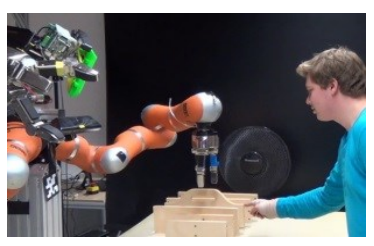

00:50:20

8: Moves hand upwards

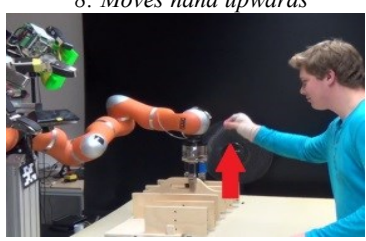

Figure 1: Example interactions: The user changes his strategy even before the robot responds

\subsection{Participants}

All 34 participants are students or employees from the technical campus at the University of Innsbruck. Their mean age is 23.9 $(\mathrm{SD}=4.57)$. There is an uneven distribution of gender so that only one third (11) of the participants are women while two thirds (23) are men.

\subsection{Robot}

The robot is an industrial robot platform equipped with a Kuka arm and a Karlsruhe humanoid head, both with seven degrees of freedom. The head was covered by a 3D-printed face to hide away the electronics and give participants an impression of a humanoid face.

\section{RESULTS}

\subsection{What do people start out with?}

The type of strategies or actions that participants start out with can be sorted into three categories: pointing gestures $(47.1 \%)$, verbal commands $(20.6 \%)$ and attention-getting gestures (such as waving a hand at the robot; $32.4 \%$ ). Actions are defined as any act, verbal or nonverbal, that participants perform with the intent of getting the robot to comply.

\subsection{What do they do next?}

The delay between participant's first and second actions is very small: 2.3 seconds $(\mathrm{sd}=1.0)$ on average. However, we did see some interpersonal variation (range $=1.01-5.11$ on a positively skewed distribution). Participants thus initiate a new action quickly after having completed their first action. Even though half of the participants $(47.1 \%)$ use a gesture that makes the robot move, only $6.3 \%$ of these realize that their instruction was successful. Instead, they perform several other actions $(m=3.09$, $\mathrm{sd}=2.43$ ) after the first one.

\subsection{How do people react to the robot}

Because participants at first do not realize that a pointing gesture will make the robot move, many of them adopt alternative strategies for how to instruct the robot. In fact, exactly half of the participants whose first action is a pointing gesture end up using their own hand to create a mirror image for the robot to follow.

\section{CONCLUSION AND IMPLICATIONS}

The study shows that the delay of the robot's response leads users to abandoning their initial instruction strategies; thus, especially those that are most intuitive to the users because they constitute their first choice are not the strategies they end up using to instruct the robot with because they interpret the robot failing to comply within two seconds as a sign that it does not understand this instruction. This inference is very similar to the interpretation of delay in human conversation, where the failure to respond within an expected timeframe (usually $300 \mathrm{msec}$ ) is understood as noncompliance with a request [8]. For the design of intuitive humanrobot tutoring interfaces this means we need to find ways in which the robot can successfully communicate that it has understood and is executing a task. How a robot can do this best is still an open question and one we will pursue in the near future.

\section{ACKNOWLEDGEMENTS}

This research was partially funded by the European Community's Seventh Framework Programme FP7/2007-2013 under grant agreement no. $610878,3^{\text {rd }}$ HAND.

\section{REFERENCES}

[1] C.L. Sidner, Lee, C.L., Kidd, C., Lesh, N. and Rich, C., Explorations in Engagement for Humans and Robots. Artificial Intelligence 166, 1-2: 140-164, 2006.

[2] H. Kose-Bagci, Dautenhahn, K., Syrdal, D.S. and Nehaniv, C.L., Drum-mate: Interaction dynamics and gestures in human-humanoid drumming experiments, Connection Science, 22(2):103-134, 2010.

[3] K.S. Lohan, K.J. Rohlfing, K. Pitsch, J. Saunders, H. Lehmann, C.L. Nehaniv, K. Fischer, and B. Wrede, Tutor spotter: Proposing a feature set and evaluating it in a robotic system. Intl. Journal of Social Robotics, 4(2):131-146, 2012.

[4] K. Fischer, Lohan, K.S., Saunders,J.,Nehaniv,C., Wrede, B., and Rohlfing, K.: The impact of the contingency of robot feedback on HRI. Cooperative Technological Systems, San Diego, May 20-24 2013.

[5] A.L. Thomaz and Breazeal,C. "Teachable robots: Understanding human teaching behaviour to build more effective robot". Artificial Intelligence 2008.

[6] M. Cakmak andThomaz,A. "Optimality of Human Teachers for Robot Learners", in the 2010 Proceedings of the $9^{\text {th }}$ IEEE Intl. Conference on Development and Learning, pp. 64-69.

[7] Chernova, S. and Thomaz, A. 2014. Robot Learning from Human Teachers. Morgan \& Claypool Publishers.

[8] S. Levinson, Pragmatics. Cambridge University Press, 1983. 